\title{
Editorial: Trends and Advanced Materials for Pavement and Road Infrastructure
}

\author{
Ramadhansyah Putra Jaya ${ }^{1 *}$, Norhidayah Abdul Hassan ${ }^{2}$ and Savas Erdem ${ }^{3}$ \\ ${ }^{1}$ Department of Civil Engineering, College of Engineering, Universiti Malaysia Pahang, Kuantan, Malaysia, ${ }^{2}$ School of Civil \\ Engineering, Faculty of Engineering, Universiti Teknologi Malaysia, Johor Bahru, Malaysia, ${ }^{3}$ School of Civil Engineering, Istanbul \\ University-Cerrahpasa, Avcilar Campus, Istanbul, Turkey
}

Keywords: waste material, highway engineering, pavement (concrete), bitumen, digital image

Editorial on the Research Topic

Trends and Advanced Materials for Pavement and Road Infrastructure

Welcome to the special issue of 2021 "Trends and Advanced Materials for Pavement and Road Infrastructure". A year with the Covid-19 pandemic has caused great impacts on all aspects of society worldwide. With the current challenges, this special issue offers four interesting technical papers comprising research on bitumen modification, pavement noise, waste material, and modeling of concrete bridges.

In the last decade, research into the alteration of road materials has progressed significantly. This is because the material used has a dominant role, particularly in determining pavement performance, and is closely related to the sustainability of road construction. It is also a significant aspect to be considered in road construction and maintenance for its long-term performance. Various research topics are available to address this issue and extend the pavement's service life with several new techniques emerging in the field of advanced materials for pavement and road infrastructure. Hence, the editorial team believes it is important to pay special attention to this issue.

In the present issue of Frontiers in Materials, two of the four articles accepted deal with the different aspects of asphalt modification, and the rest cover pavement modelling.

The first paper by Suo et al. addressed the applicability of waste corn oil and soybean oil in the performance recovery of aged bitumen in recycled asphalt mixture. The materials were tested for physical properties, viscosity, and detailed rheological characteristics. It was concluded that the vegetable oil effectively reduces the viscosity and stiffness of the aged bitumen and improves fatigue and low temperature cracking resistance. The findings show improvement in the bitumen recovery with a recommendation that between 6 and $8 \%$ vegetable oil is suitable to be used in mixing with the aged bitumen.

In recent years, the proliferation of plastic waste has become a global problem. In line with the aim of this research topic, Radeef et al. highlighted the effectiveness of using plastic waste as an additive in the asphalt mixture. Crushed granite aggregate with a size of $14 \mathrm{~mm}$ and shredded plastic waste in the range of 5-10 mm were applied in the study. The authors concluded that the addition of plastic waste using the dry process leads to deviation in the aggregate structure (high plastic content). Asphalt mixtures modified with $1 \%$ plastic waste via dry method showed significant enhancement in the asphalt pavement performance.

Preventive maintenance techniques for asphalt pavements such as micro-surface (MS) have the advantages of waterproofing and crack sealing. Chen et al. presented their study on the surface texture of pavements with low-noise micro-surface using three-dimensional imaging technology. In the investigation, five low-noise MS were systematically analyzed and compared based on surface texture and noise. A three-dimensional digital texture model (3D-DTM) of MS surface texture was 
constructed using a series of digital image processing techniques. The authors found that the digital image processing techniques are very helpful in analyzing the pavement texture characteristics and are also a promising tool to optimize the design of low-noise MS.

Time-dependent responses of cracked structures are complex due to the intertwined effects between creep-shrinkage and cracking. Our final paper highlights the lack of an effective numerical model to accurately predict the nonlinear pattern of long-term deflections. Therefore, Tong et al. proposed the gradient nonlocal enhanced micro pre-stress-solidification theory with finite elements. From the proposed model, they found that the proposed model reflects the creep and shrinkage properties of concrete with respect to underlying moisture transport and heat transfer.

In general, we hope that the detailed research presented in this special issue provides a better understanding and interest to all researchers and practitioners who are focused on the recent developments and advancements in analyzing pavement performance and road infrastructure.

\section{AUTHOR CONTRIBUTIONS}

All authors listed have made a substantial, direct, and intellectual contribution to the work and approved it for publication.

Conflict of Interest: The authors declare that the research was conducted in the absence of any commercial or financial relationships that could be construed as a potential conflict of interest.

Publisher's Note: All claims expressed in this article are solely those of the authors and do not necessarily represent those of their affiliated organizations, or those of the publisher, the editors and the reviewers. Any product that may be evaluated in this article, or claim that may be made by its manufacturer, is not guaranteed or endorsed by the publisher.

Copyright (c) 2021 Putra Jaya, Abdul Hassan and Erdem. This is an open-access article distributed under the terms of the Creative Commons Attribution License (CC BY). The use, distribution or reproduction in other forums is permitted, provided the original author(s) and the copyright owner(s) are credited and that the original publication in this journal is cited, in accordance with accepted academic practice. No use, distribution or reproduction is permitted which does not comply with these terms. 